\title{
A paper-based microfluidic platform with shape- memory-polymer-actuated fluid valves for automated multi-step immunoassays
}

Hao Fu (10) ${ }^{1,2}$, Pengfei Song ${ }^{1,2,3}$, Qiyang Wu ${ }^{1,2}$, Chen Zhao ${ }^{1}$, Peng Pan ${ }^{1,2}$, Xiao Li ${ }^{2,6}$, Nicole Y. K. Li-Jessen ${ }^{4}$ and Xinyu Liu (1) ${ }^{1,5}$

\begin{abstract}
Smart fluid manipulation with automatically controlled paper valves will enable automated and multi-step immunoassays on paper-based microfluidic devices. In this work, we present an integrated paper-based microfluidic platform with shape-memory polymer (SMP)-actuated fluid valves capable of automated colorimetric enzyme-linked immunosorbent assays (ELISAs). A single-layer microfluidic paper-based analytical device ( $\mu$ PAD) was designed to store all the reagents on the chip, and sequentially transfer reagents to a paper test zone following a specific ELISA protocol through automatic fluidic flow control by the multiple SMP-actuated valves. The actuation of a paper valve was based on the thermally responsive, duel-state shape transformation of a SMP sheet attached to the root of a paper cantilever beam for driving a hydrophilic paper bridge to connect and disconnect two paper channels. A portable colorimetric reader was developed to control the on-chip valve operations, quantify the colorimetric signal output, display the assay result, and wirelessly transmit the data to a smart phone for the application of telemedicine. Reliable operations of the paper valve and the entire $\mu \mathrm{PAD}$ were demonstrated with success rates of $97 \%$ and $93 \%$, respectively. A detection mechanism for valve malfunction was designed and confirmed effective to identify any mal-operation of individual valves, thus rendering our platform reliable in real assays. For device calibration, we conducted direct ELISAs of rabbit lgG in phosphate-buffered saline (PBS), and achieved a low limit of detection (LOD) of $27 \mathrm{pM}$ (comparable to that of standard and paper-based ELISAs). In order to demonstrate the clinical application of our multi-step immunoassay platform, we also conducted sandwich ELISAs to quantify the protein level of an inflammatory cytokine, namely tumor necrosis factor (TNF)-a, in surgically injured laryngeal tissues of rats. The protein levels of TNF-a were shown similar between the conventional and $\mu$ PAD ELISAs.
\end{abstract}

\section{Introduction}

Point-of-care testing (POCT) is designed for rapid diagnostic assays with satisfactory accuracy and sensitivity, low sample/reagent consumptions, and excellent cost efficiency. POCT has enabled effective healthcare in resource-limited settings, and has supplemented or

\footnotetext{
Correspondence: Xinyu Liu (xyliu@mie.utoronto.ca)

'Department of Mechanical and Industrial Engineering, University of Toronto, Toronto, ON, M5S 3G8, Canada

${ }^{2}$ Department of Mechanical Engineering, McGill University, Montreal, QC, H3A OC3, Canada

Full list of author information is available at the end of the article.
}

replaced the conventional diagnostics of existing healthcare systems. Microfluidic paper-based analytical devices ( $\mu$ PADs) represent one of the most promising platform technologies for POC diagnostics, in which paper substrates are employed to bring various merits to analytical tests ${ }^{1}$. Many types of bioassays have been implemented on the $\mu$ PADs by colorimetry ${ }^{2-4}$, fluorometry ${ }^{5}$, electrochemistry $^{6}$, and electrochemiluminescence ${ }^{7}$ for detection of proteins based on binding of reporters (e.g., ionized color dyes and enzyme-conjugated antibodies) to target analytes. Among a diverse range of bioassays, the enzymelinked immunosorbent assay (ELISA) is a gold standard of

\section{(c) The Author(s) 2019}

(c) (i) Open Access This article is licensed under a Creative Commons Attribution 4.0 International License, which permits use, sharing, adaptation, distribution and reproduction in any medium or format, as long as you give appropriate credit to the original author(s) and the source, provide a link to the Creative Commons license, and indicate if changes were made. The images or other third party material in this article are included in the article's Creative Commons license, unless indicated otherwise in a credit line to the material. If material is not included in the article's Creative Commons license and your intended use is not permitted by statutory regulation or exceeds the permitted use, you will need to obtain permission directly from the copyright holder. To view a copy of this license, visit http://creativecommons.org/licenses/by/4.0/. 
protein detection in clinical samples associated with diseases $^{3,4}$. However, in most of the existing $\mu$ PAD designs running ELISAs ${ }^{3,8}$, the multi-step assays were performed manually. The assay process involves human operations such as repeated pipetting of samples/reagents and quantification of the assay results using imaging devices such as scanner, camera, and microscope. Often, the user needs to operate the $\mu \mathrm{PAD}$ by following a specific protocol. Thus, the inability of these $\mu$ PADs to autonomously carry out the entire ELISA process limits, to some extent, their applications to certain diagnostic scenarios where some tests would need to be conducted in a 'sample-inresult-out' (SIAO) fashion.

Although the capillary wicking in a porous paper substrate of $\mu$ PADs eliminates the requirement of external pumps for driving fluids to perform assays, controlled fluid manipulation in porous paper channels is not as straightforward as in the conventional hollow microfluidic channels. A variety of fluid manipulation strategies on $\mu$ PADs have been developed for achieving on-chip fluid control at certain levels, including mechanical valves ${ }^{9-12}$, channelgeometry-based fluid regulation ${ }^{13,14}$, fluidic diodes ${ }^{15-17}$, dielectric electrowetting ${ }^{18}$, dissolvable bridges ${ }^{19,20}$, meltable wax valves ${ }^{21,22}$, porous shunt ${ }^{23}$, paper carving ${ }^{24}$, selectively permeable barrier $^{25}$, and electrostatic control ${ }^{26}$. Benefitting from these fluid manipulation strategies, human operation is no longer a necessity for fluid regulation on a $\mu \mathrm{PAD}$. Among these fluid manipulation methods, the mechanical valves are straightforward in terms of device design, and primarily rely on the control of connection and disconnection between channels. For instance, a paper cantilever beam was operated manually to control the connection and disconnection of two channels ${ }^{9}$. Sliding operation can bring channels in different paper layers into contact to transfer fluids and run multi-step assays ${ }^{4,10,27}$. Push-button valves were designed for manual compression to bridge a gap between two channels in different layers of a $\mu \mathrm{PAD}$ for fluid transport ${ }^{11}$. Besides valve designs in devices with hydrophilic paper channels, $\mu$ PADs with embossed hollow channels also involved a valving mechanism through folding and unfolding of the paper substrate to turn on and off fluid flows in the hollow channels ${ }^{12}$. One common drawback of the aforementioned valve designs is the requirement of manual operations.

Automated operations of mechanical valves on $\mu$ PADs were also demonstrated, making autonomous on-chip assays possible. We previously reported a magnetic timing valve for timed fluid control on $\mu \mathrm{PADs}^{28}$. This design functionalized a paper cantilever beam with magnetic nanoparticles, and used an electromagnet to actuate the paper beam for one-way connection or disconnection of a paper channel. The operation of each paper beam valve, however, requires a separate electromagnet. For a $\mu \mathrm{PAD}$ with multiple magnetic valves, the required electromagnets need to be separated from each other with a sufficient spacing to avoid mal-manipulation of paper valves caused by magnetic cross-talks. Also, the same electromagnet can only activate or deactivate its corresponding valve, i.e., one-way operation. On the other hand, Thuo and co-workers proposed a two-way operation design of magnetic paper valves ${ }^{29}$. Hard magnets were patterned on one end of a paper cantilever valve through impregnation of hard magnetic polymer, allowing the valve to be magnetically attracted or repelled by a magnet and thus operate at three different positions, i.e., the neutral, on, and off positions. However, similar to our previous design ${ }^{28}$, each valve still needs an electromagnet for actuation, and the relatively large size of the electromagnet and the required large spacing of adjacent electromagnets (for avoiding valve cross-actuation) limit the integration density of these valves on a $\mu$ PAD. Yager and co-workers demonstrated a valve activation method on $\mu$ PADs with compressed sponges as localized actuators in an integrated diagnostic toolkit ${ }^{30}$, and performed ELISAs for SIAO testing. However, the device includes many moving parts (e.g., test strips, sponge-based valves, and glass fiber actuation channels), making its usage less straightforward and its manufacturing potentially challenging. For continuous testing of multiple samples, the loading of a new $\mu$ PAD may need assembly of a paper strip and its moving parts, thus lowering the operation efficiency. Furthermore, the toolkit only provides qualitative diagnostic answers directly, and subsequent analysis by a professional is required for obtaining quantitative results.

In this paper, we present a new design of thermallyactivated paper valves that enables the development of a fully-automated paper-based microfluidic device for performing colorimetric ELISAs in a SIAO fashion. The thermally responsive shape-memory polymer (SMP) was used, for the first time, to actuate a paper cantilever beam on a $\mu \mathrm{PAD}$ for activation and deactivation of a paper valve. A unique feature of our valve design is that the same SMP actuator is capable of both turning on and off a valve, which is based on the due states (temporary and permanent shapes) of the SMP material. We designed a $\mu \mathrm{PAD}$ that stores all the required reagents on a chip and sequentially transports individual reagents to a test zone of the device to enable a multi-step ELISA. We also developed a portable colorimetric reader to control the on-chip paper valves for automatically conducting an ELISA and to quantify the colorimetric output. To run an assay, a user only needs to mount a $\mu \mathrm{PAD}$ onto the platform, add the sample and reagent-transferring buffer to the $\mu \mathrm{PAD}$, and wait until the assay is completed and the result is displayed on the device screen. The platform typically completes a direct ELISA within $55 \mathrm{~min}$. We also proposed a novel self-checking mechanism for reliably 
monitoring valve malfunction, which is based on detection of the light-transmittance differences of the test zone in wet versus dry states. By using this platform, we performed direct ELISA of rabbit IgG in phosphate-buffered saline (PBS) and sandwich ELISA of tumor necrosis factor (TNF)- $\alpha$, an inflammatory cytokine marker, obtained from rat laryngeal tissue samples, and achieved high analytical performance that is comparable to that of standard ELISA performed on a 96-well plate.

\section{Results and discussion}

\section{The $\mu$ PAD with SMP-actuated valves}

Figure 1a schematically illustrates the design of a $\mu \mathrm{PAD}$ with four SMP-actuated paper valves to run a direct ELISA. All reagents required by the ELISA are stored (in a dry form) in the storage zones ( $6 \mathrm{~mm}$ in diameter) that are all connected, through the paper valves, to a central test zone ( $6 \mathrm{~mm}$ in diameter). To carry out a test, a sample solution is first added to the test zone and five drops (total volume: $250 \mu \mathrm{L}$ ) of $1 \times$ PBS buffer to the device inlet with a syringe. Then, individual reagents are transferred to the test zone sequentially in a pre-defined order, and different valves are activated accordingly to allow the PBS to transfer the reagents to the test zone. The SMP-actuated valves are automatically controlled by a colorimetric reader (on which the $\mu \mathrm{PAD}$ is operated) to switch on and off, and the reader determines the sequence and timing of reagent transfers based on a pre-programmed ELISA protocol.

Thermally responsible SMPs have been used to fabricate actuators of origami robots to achieve localized joint rotation and three-dimensional folding/unfolding ${ }^{31,32}$. A piece of thermally responsive SMP can deform from its permanent shape to a temporary shape when heated to a temperature above its switching transition temperature $\left(T_{\text {trans }}\right)$, and then maintain its temporary shape when cooled down to a temperature below $T_{\text {trans }}$. When heated up again above $T_{\text {trans }}$, the SMP will transform back to its permanent shape. Based on this dual-state property, we introduced the thermally responsive SMP in our device design for two-way actuation of the paper valve, thus enabling automatic fluid control on a $\mu \mathrm{PAD}$ for running multi-step assays.

As schematically shown in Fig. 2a, a sheet of polyolefin $\left(\mathrm{PO} ; T_{\text {trans }}=95^{\circ} \mathrm{C}\right)$ with a curved permanent shape was

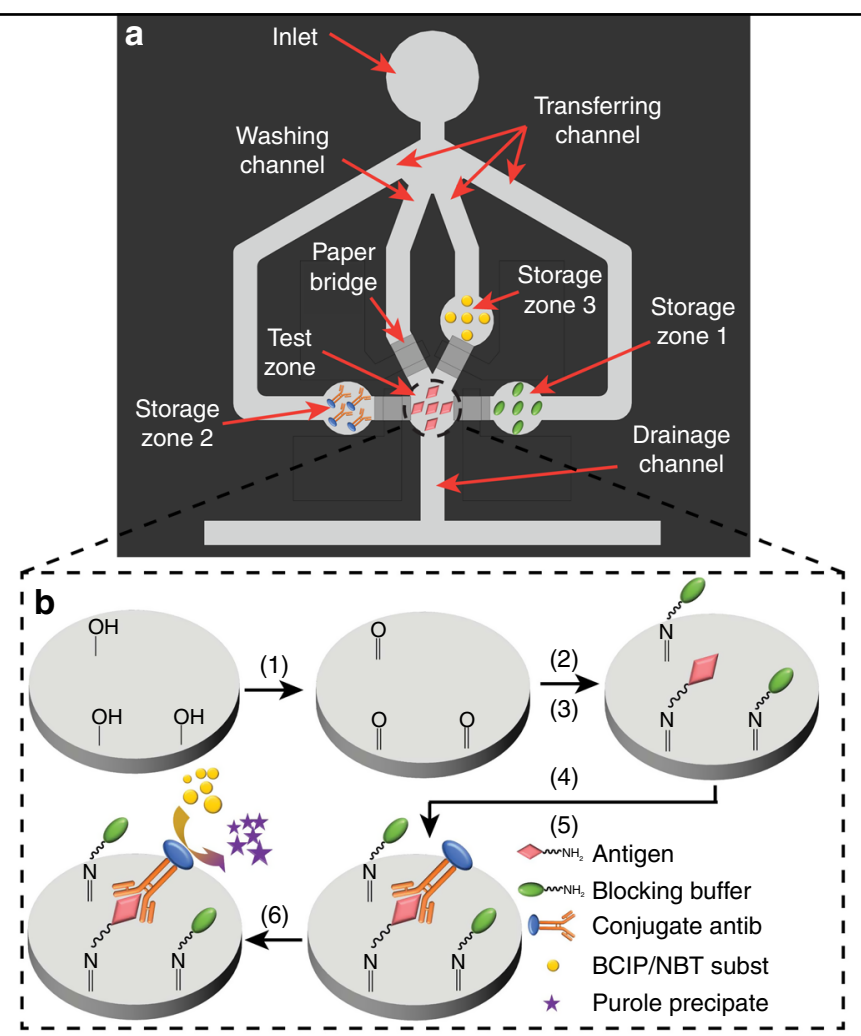

Fig. 1 Schematic diagrams of the $\mu$ PAD design and the direct ELISA protocol. a Schematic top view of a $\mu$ PAD integrating four SMP-actuated valves (the valves are attached to the back of the $\mu P A D$ ) and $\mathbf{b}$ The schematic protocol of a direct ELISA that can be performed in the test zone of the MPAD. The protocol of a direct ELISA is carried out in six steps: (1) modifying the paper test zone surface with aldehyde groups using potassium periodate $\left(\mathrm{KIO}_{4}\right)$; (2) immobilizing antigens on the modified paper through covalent bonding; (3) blocking the test zone surface with blocking buffer; (4) labeling antigens with enzyme-conjugated antibodies; (5) washing away unbound antibodies; and (6) adding enzyme substrate for colorimetric signal production 

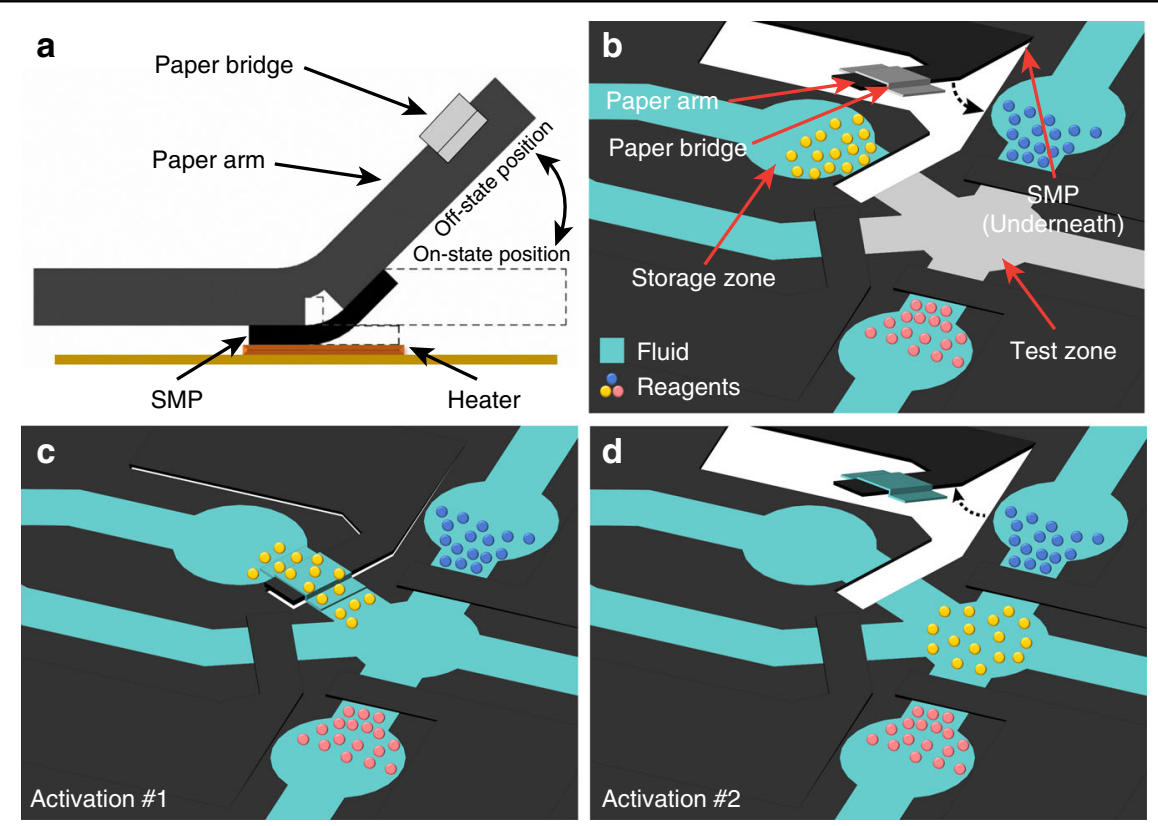

Fig. 2 Principle and operation of the SMP-actuated valve on the $\boldsymbol{\mu P A D} . \mathbf{a}, \mathbf{b}$ Side and oblique views of a paper cantilever arm that is laser-cut out of a single-layer $\mu$ PAD and attached with a SMP-actuated valve at its root. The initial state of a valve is bent up. c The SMP is first activated to reach its temporary flat shape ("activation \#1"), which bends down the paper arm and makes the paper bridge connect the reagent storage zone and the test zone for reagent transfer. $\mathbf{d}$ The SMP is then activated again to recover its permanent curved shape ("activation \#2"), which disconnects the reagent storage zone and the test zone and stops the transfer process. The dashed lines indicate the actuation paths of the paper cantilever beams for activation \#1 and \#2

attached to the root of a paper cantilever arm (which is laser-cut out of a single-layer $\mu \mathrm{PAD}$ ), and used as a bending SMP actuator. The initial bending angle of the paper arm was $30^{\circ}$. A copper heating resistor, patterned on a printed circuit board (PCB), was arranged underneath the SMP to activate the valve through joule heating ${ }^{31}$. When heated above $95^{\circ} \mathrm{C}$, the SMP lowers the paper beam to its on-state position (Fig. 2c; which is called "activation \#1"), and can maintain this position even the heating is removed. When heated above $95^{\circ} \mathrm{C}$ again, the SMP raises the paper arm back to its off-state position (Fig. 2d; "activation \#2"). A hydrophilic paper bridge attached to the end of the paper arm connects the reagent storage zone and the test zone when the valve is turned on.

We experimentally determined in-plane size of the PO sheet and its heating resistor underneath to be $6 \mathrm{~mm} \times$ $12 \mathrm{~mm}$, which was large enough to ensure efficient heating and activation of the PO sheet. With the selected inplane dimension of the PO sheet, we also compared the operation success rate of the paper valves made from three different types of commercially available PO sheets, and eventually chose the one (RNF-100 $1^{\prime \prime} \times 4^{\prime}$ BLK, TE Connectivity) with a thickness of $0.89 \mathrm{~mm}$ as it provided the highest activation success rate and appropriate response time (Table S1). With the final designs of the SMP valve and the heating resistor, the minimal heating time required for activations \#1 and \#2 was measured to be $22.7 \pm 3.7 \mathrm{~s}(n=15)$ and $24.4 \pm 5.3 \mathrm{~s}(n=15)$, respectively (Table S1). Note both time periods were measured with the SMP valve initially at room temperature. To reliably activate the SMP valve to turn on and off, we set a fixed heating time at $35 \mathrm{~s}$ for both activation steps of \#1 and \#2. During operation of the SMP valve, no temperature rise was measured in the paper test zone.

\section{Integration of the $\mu \mathrm{PAD}$ with a colorimetric reader}

To automatically run an ELISA and measure its colorimetric output, we developed a portable colorimetric reader (Fig. 3a) to host the $\mu$ PAD. The reader includes three major parts: (i) an operation cell for automatic operation of the on-chip paper valves and accurate measurement of the output signal, (ii) a microcontroller circuit to control the operation cell, and (iii) a Bluetooth module for data transmission to a cellphone or a computer. In the operation cell, a PCB with patterned copper heating resistors is arranged underneath the $\mu \mathrm{PAD}$ to activate the SMP actuators. The heating resistors were patterned by wet etching of the copper layer of a coppercoated polyimide sheet. For maximizing the heating efficiency, the copper traces of each heating resistor were patterned in a serpentine shape of $0.5 \mathrm{~mm}$ wide ${ }^{31}$, and the overall size of the resistor was designed to be the same $(6 \mathrm{~mm} \times 12 \mathrm{~mm})$ as the SMP sheet size of the valve. The resistance of the heating resistor was $0.3 \Omega$, and heating 


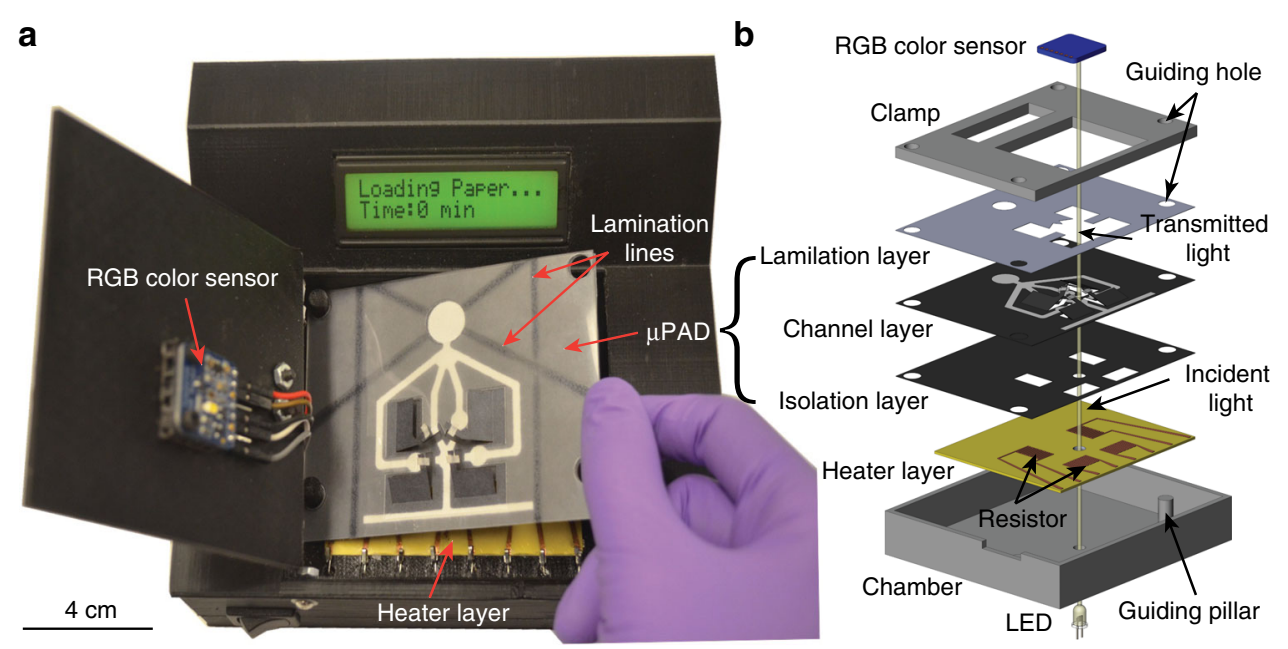

Fig. 3 Integration of the $\mu$ PAD with a colorimetric reader. a Photograph of the colorimetric reader that accommodates a $\mu$ PAD for activation of the valves and readout of the colorimetric signal. $\mathbf{b}$ Exploded view of the combined architecture of the $\mu$ PAD and the operation cell of the colorimetric reader

current was $2 \mathrm{~A}$. Each resistor was switched on and off by a $1.2 \mathrm{~W}$ transistor controlled by the microcontroller. To integrate with the colorimetric reader, an isolation layer (made from wax-impregnated paper) with laser-cut openings (Fig. 3b) was attached to the bottom surface of the paper channel layer with double-sides tapes, which only exposes SMP sheets of the valves to the copper heater and avoids fluid leakage from the paper channel layer to the heating PCB. A plastic lamination layer (Fig. 3b) was attached on the top surface of the paper channel layer to accelerate fluid flowing rate and prevent the evaporation of the buffer. The three layers of the $\mu \mathrm{PAD}$ were assembled by thermal lamination.

To quantify the colorimetric readout, a white LED $\left(\lambda_{\max }=550 \mathrm{~nm}\right)$ on the bottom of the operation cell was used to illuminate the test zone of the $\mu \mathrm{PAD}$ when the last signal amplification step of the colorimetric ELISA was completed. A red-blue-green (RGB) color sensor (TCS34725, Adafruit) was arranged on the other side of the test zone to measure the light transmission through the test zone. The centers of the LED, the RGB sensor, and the test zone were aligned coaxially. A higher colorimetric signal level of the ELISA causes a lower level of light transmission through the test zone. The RGB sensor directly provides a digital electrical signal to the microcontroller, and the mean grayscale intensity [calculated from the RGB intensity from the sensor by taking the average of the three channel intensities: grayscale $=(\mathrm{R}+$ $\mathrm{G}+\mathrm{B}) / 3$ ] is displayed on the LCD screen as the final result. The test results can also be transmitted to a cellphone or computer through Bluetooth communication. By pre-programming a specific ELISA protocol (i.e., timings of valve operations and colorimetric signal readout) in the microcontroller, and automatic ELISA can be achieved on the platform in a SIAO fashion. To run a test, a user only needs to load a $\mu$ PAD to the reader, add a drop of sample to the test zone and several drops $(\sim 250 \mu \mathrm{L})$ of PBS buffer to the device inlet, then close the door and wait for a period of time before the result is displayed.

Figure S1 illustrates the graphic user interface (GUI) of Bluetooth communication application (APP) on an Android-based cellphone (Xperia ${ }^{\mathrm{Tm}} \mathrm{Z}, \mathrm{SONY}$ ). The Bluetooth module can transmit assay results to the cellphone, and the cellphone APP can display the data graph and further transmit the results to a remote site through text message. The cellphone APP can control the Bluetooth port of the platform, trigger the automated assays, and receive the data via Bluetooth transmission.

\section{Determination of the operation parameters}

Before calibrating the device performance, we first experimentally determined two major operation parameters, namely, (i) the reagent transfer time: the necessary time required for transferring an on-chip stored reagent from its storage zone to the test zone (i.e., the on-state period of the valve) under continuous flow of PBS buffer; and (ii) the test zone washing time: the time required for removing unbound antibodies that are added to the test zone to bind with the target antigen in direct or indirect ELISA under continuous PBS flow. To determine the reagent transfer time, we performed reagent transfer experiments on the $\mu \mathrm{PAD}$ using fluorophore-conjugated rabbit IgG antibody (anti-rabbit IgG). First, we spotted $3 \mu \mathrm{L}$ of fluorescein isothiocyanate (FITC)-conjugated anti-rabbit IgG solution $(0.1 \mathrm{mg} / \mathrm{mL})$ and $3 \mu \mathrm{L}$ of blocking buffer solution $[0.5 \%(\mathrm{v} / \mathrm{v})$ Tween- 
20 and $10 \%(\mathrm{w} / \mathrm{v})$ BSA in PBS] on the storage zones, and waited for $10 \mathrm{~min}$ at room temperature to dry the test zone. Then, we added $\sim 250 \mu \mathrm{L}$ of PBS to the inlet using a syringe. Once the PBS flow passed the storage zone and reached the paper valve (which was initially in its offstate), the valve was switched on to allow the PBS flow to transfer the FITC-conjugated anti-rabbit IgG to the test zone. The regent transfer time was counted as the time period during which the valve was on and the reagentcarrying PBS solution was flowing through the test zone. Once the valve was turned off, the test zone was incubated at ambient environment for $10 \mathrm{~min}$. Finally, the FITC fluorescence intensity of the test zone, which corresponded to the amount of anti-rabbit IgG transferred to the test zone, was quantified by a fluorescence microscope (SZX-16, Olympus; excitation: $490 \mathrm{~nm}$, and emission: $520 \mathrm{~nm}$ ) with a cooled fluorescence camera (EXi Blue, QImaging). We found that the measured FITC intensity plateaued after a transfer time of $45 \mathrm{~s}$ (Fig. S2a). Thus, after the $35 \mathrm{~s}$ heating for activation \#1 was completed, we set a $30 \mathrm{~s}$ waiting time before starting activation \#2 to cool down the SMP valve to room temperature. As the heating time for activation $\# 2$ is $24.4 \pm 5.3 \mathrm{~s}$ (before which the valve was on), the total reagent transfer time (average: $30+24.4$ $=54.4 \mathrm{~s}$ ) was found to be sufficient to achieve the maximum transfer efficiency.

With regard to the test zone washing time, we added $3 \mu \mathrm{L}$ of FITC-conjugated anti-rabbit IgG directly to a test zone (without surface functionalization), incubated it for $1 \mathrm{~min}$, and used the washing channel (Fig. 1a) to wash the test zone by continuous PBS flow from the inlet. Since the test zone was not aldehyde-functionalized, the added antirabbit IgG only formed weak physical bonding (similar to non-specific binding in an ELISA) with the surfaces of cellulose microfibers in the test zone, and can, in principle, be completely washed off under thorough perfusion. We quantified the residual FITC intensities of the test zones that had been washed for four different time lengths $(30,90,150$, and 210 s). From Fig. S2b, once can see that after $150 \mathrm{~s}$ washing, more than $90 \%$ of the non-specific binding in the test zone was removed. Accordingly, for activating the washing valve, the waiting time between activation \#1 and \#2 was set to be $130 \mathrm{~s}$ (average time for washing: $130+24.4=154.4 \mathrm{~s}$ ).

\section{Success rate of valve operation and self-checking mechanism for valve malfunction}

Video S1 shows the loading of a $\mu$ PAD onto the colorimetric reader, and Video S2 illustrates the operation of four SMP-actuated valves on a $\mu$ PAD for direct ELISA. In Video S2, colored dyes were used to mimic the ELISA reagents and were pre-stored in the reagent storage zones for visualization of the reagent transfer. Note that the door of the colorimetric reader, which should be always closed during a real assay, was opened for taking Video S2. We operated $100 \mu \mathrm{PADs}$ of this kind (which include 400 SMP-actuated valves in total) to quantify the success rates of valve operation and device operation. Among the 400 valves, 388 valves operated normally, corresponding a success rate of valve operation at $97 \%$. We counted the operation of a $\mu \mathrm{PAD}$ as a failure if any of its four valves malfunctioned, and obtained a success rate of device operation at $93 \%(n=100)$. To further improve the device operation success rate, other SMP materials with different thicknesses could be investigated.

Despite the high success rate of valve operation, it is still highly desired to detect the malfunction of a specific valve in the practical use of a $\mu \mathrm{PAD}$. To this end, we established a self-checking mechanism for valve malfunction through detecting the wetting condition of the test zone. We used the LED and RGB sensor pair to measure the transparency because a wet paper is more transparent than a dry paper. If a valve fails to switch on (activation \#1 fails), no PBS buffer will be transferred to the test zone and a lower transparency of the test zone will be measured. Similarly, the failure of a valve during activation \#2 (switching off) will cause the PBS still to flow through the test zone, thus induce a test zone transparency higher, after 1-min waiting, than normal. Thus, by detecting the light transmittance through the test zone right after a valve was switched on (activation \#1) or $1 \mathrm{~min}$ after a valve was switched off (activation \#2), the colorimetric reader was capable of detecting any valve malfunction with a detection success rate of $100 \%$.

For the direct ELISA protocol (Fig. 1b), we carried out reagent transfer experiments and measured the mean grayscale intensities of the light transmitted through the test zone before and after activations \#1 and \#2 for each valve of the $\mu \mathrm{PAD}$ (results shown in Fig. 4). For activation $\# 1$ of the valve, the grayscale intensities were measured right before the activation \#1 was initiated (i.e., heating resistor was turned on) and $10 \mathrm{~s}$ after the $35 \mathrm{~s}$ heating for activation \#1 was completed. For activation \#2 of the valve, the grayscale intensities were measured right before the activation \#2 was initiated and 1 min after the $35 \mathrm{~s}$ heating for activation \#2 was completed. From Fig. 4, one can see that the grayscale intensity varied significantly from its normal value if a valve failed during activations \#1 and \#2, allowing the reliable detection of any valve malfunction during an assay. Once a valve malfunction is detected, the colorimetric reader stops the ongoing assay and reminds the user of the failure. As we obtained a high device operation success rate of $93 \%$, statistically only $7 \%$ of $\mu$ PADs may fail during operation. This $7 \%$ of failed devices were all detected with this selfchecking mechanism. 


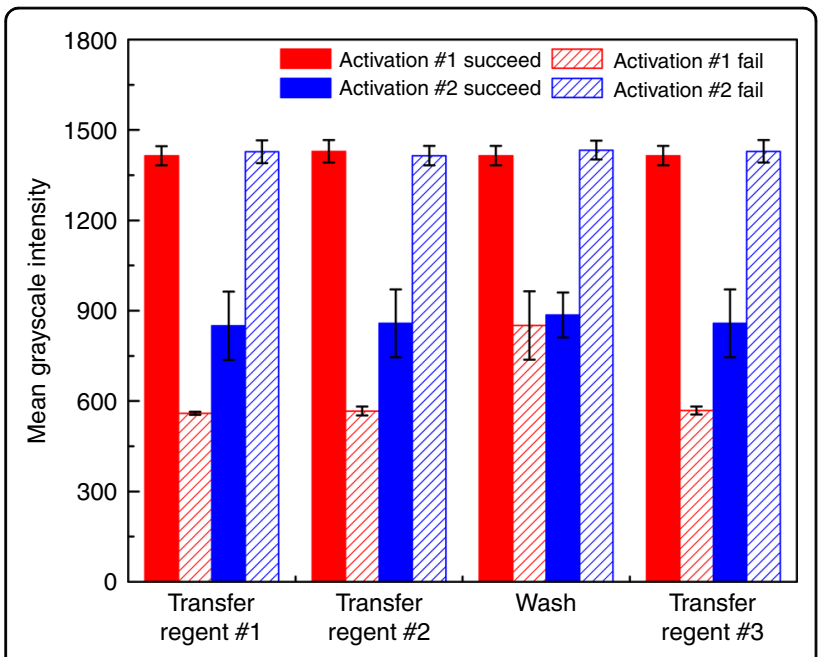

Fig. 4 Light-transmittance signals $(n=5)$ measured from the test zone at each step of the direct ELISA (Fig. 1b), right after the valve is in activation \#1 and $1 \mathrm{~min}$ after the valve is in activation \#2. Reagents \#1, \#2, and \#3 are the blocking buffer, the enzymeconjugated antibody, and the enzyme substrate. When the valve activation fails to work after each step, the measured lighttransmittance signals of the test zone are different from the normal state based on the unusual wetting conditions, enabling the detection of the valve malfunction

\section{Automatic direct ELISA of rabbit IgG antigen in PBS}

As a proof of demonstration, we carried out automated direct ELISA for the detection of rabbit IgG on our platform. Before each experiment, the following reagents were stored on the $\mu$ PAD: (i) $3 \mu \mathrm{L}$ of blocking buffer of $0.5 \%(\mathrm{v} / \mathrm{v})$ Tween-20 and $10 \%(\mathrm{w} / \mathrm{v})$ BSA in PBS, (ii) $3 \mu \mathrm{L}$ of PBS solution of the alkaline phosphatase (ALP)-conjugated IgG antibodies $(0.1 \mathrm{mg} / \mathrm{mL})$, and (iii) $3 \mu \mathrm{L}$ of 5 bromo-4-chloro-3-indolyl phosphate and nitro blue tetrazolium substrate $(4.59 \mathrm{mM} \quad \mathrm{BCIP}, 3.67 \mathrm{mM}$ NBT, $50 \mathrm{mM} \mathrm{MgCl}_{2}$ in $1 \mathrm{M}$ Tris buffer, $\mathrm{pH}$ 9.5). Then, we waited for $10 \mathrm{~min}$ at room temperature to dry the storage zones. If long-time storage of the prepared $\mu \mathrm{PAD}$ is needed before an assay, one could storage the device in an air-tight bag with desiccator and also added protein stabilizer to prevent the degradation of the stored reagents ${ }^{8}$.

To run a test, we mounted a $\mu \mathrm{PAD}$ onto the colorimetric reader, added $3 \mu \mathrm{L}$ of the rabbit IgG solution to the test zone and $\sim 250 \mu \mathrm{L}$ of PBS washing buffer to the $\mu$ PAD inlet, and then initiated the assay. The platform was preprogrammed to automatically control individual valves and carry out the following steps of the assay. (i) The blocking buffer was transferred to the test zone 3 min after the assay was started (the 3-min waiting time allows the washing buffer to travel from the inlet to the individual valves), and incubated for $10 \mathrm{~min}$. (ii) The ALPconjugated antibody was then transferred to the test zone for labeling the immobilized antigens, and incubated for $1 \mathrm{~min}$. (iii) The valve connecting the washing channel and the test zone was switched on to wash the test zone for $2.5 \mathrm{~min}$ and remove any unbound antibodies. After the washing valve was switched off, the operation paused for 10 min to drain the test zone. (iv) The BCIP/NBT substrate was transferred the test zone, and incubated for $30 \mathrm{~min}$ for signal amplification. (v) The pair of LED and the RGB color sensor was finally initiated for quantifying the colorimetric signal. Note that all the assay steps were performed at room temperature. The assay result was expressed as the mean grayscale intensity calculated from the raw RGB outputs from the RGB color sensor. The result was directly displayed on the LCD of the colorimetric reader for data recording. During the assay, the self-checking mechanism for valve malfunction constantly monitored the valve operations. Once a valve malfunction occurred, the platform reported an error message on the $\mathrm{LCD}$ and reminded the user to replace the $\mu \mathrm{PAD}$.

We carried out direct ELISA of rabbit IgG in 10-fold dilutions (6.7 pM to $6.7 \mu \mathrm{M})$, and the calibration data of our platform was shown in Fig. 5a. We also captured the test zone image after each assay with a desktop scanner (CanoScan LiDE 210, CANON Inc., setting: color photo scanning, 300-dpi resolution) and a cellphone camera Xperia $^{\mathrm{Tm}}$ Z, SONY Electronics Inc., image size: $4128 \times$ 3096 pixels). For the cellphone, we took the test zone images inside a mini photo studio (Mini Portable Photo Studio Shooting Tent, JHS-TECH) with uniform illumination, which minimized the illumination variations of the ambient environment. We measured the mean grayscale intensities of all the test zones using ImageJ by the same average method for the colorimetric reader. All the data sets were fitted into sigmoidal curves ( $s$-curves in Fig. 5b) using the Hill equation ${ }^{3}$ (Eq. S1), from which the limit of detection (LOD) and the coefficient of determination (COD; denoted as $R^{2}$ ) for each $s$-curve were calculated.

The calculated LODs and CODs are LOD $=27 \mathrm{pM}$ and $R^{2}=0.993$ for the RGB color sensor, LOD $=255 \mathrm{pM}$ and $R^{2}=0.970$ for the scanner, and LOD $=836 \mathrm{pM}$ and $R^{2}=$ 0.894 for the cellphone. The LOD of our platform for rabbit IgG detection is comparable to that (18 pM) of the previously reported paper-based ELISA ${ }^{3}$. Despite the relatively uniform illumination provided by the mini photo studio, the mean grayscale intensities of the test zones measured by the cellphone camera were still subject to larger variations because of the ISO self-adjustment of the cellphone camera when taking a photo. Therefore, the cellphone provided the lowest signal-to-noise ratio (SNR; which is defined as the ratio of the average to the standard deviation of the intensity data at each concentration) among the three colorimetric measurement methods (Fig. S3), and thus the highest LOD and the lowest COD. The scanner employed uniform scanning illumination in an enclosed flatbed, making the SNR of its calibration curve 


\section{a}

Test zone

Rabbit lgG
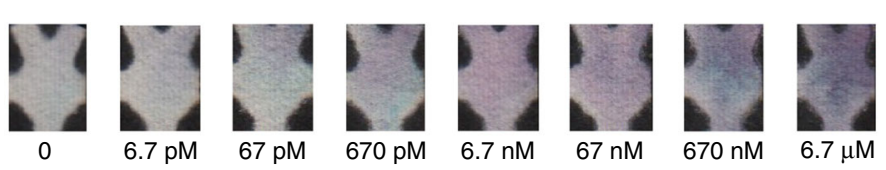

\section{b}
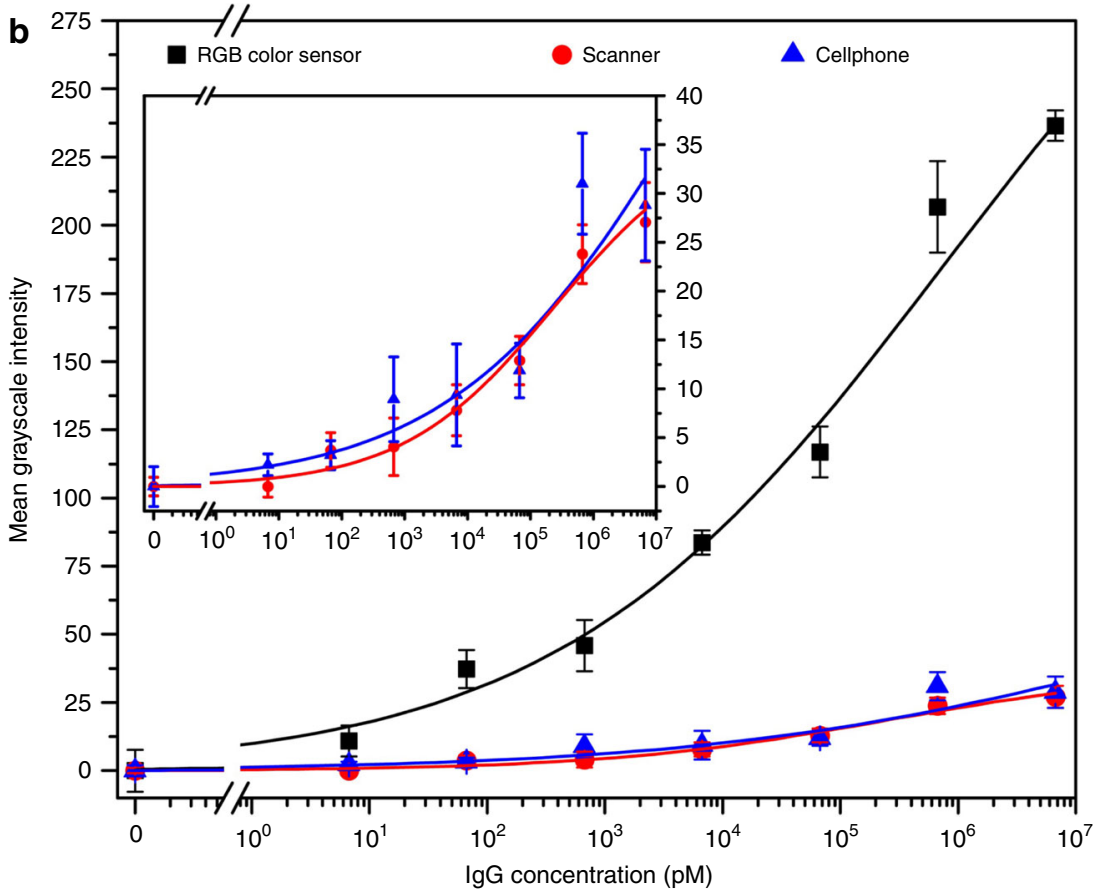

Fig. 5 Results of direct ELISA on the $\mu$ PAD for detection of rabbit IgG in PBS. a Photographs of test zones at different lgG concentrations. $\mathbf{b}$ Calibration curves of the mean grayscale intensity signal versus the $\lg G$ concentration on the test zone $(n=5)$. Inset: amplification of the low mean grayscale intensity range (from 0 to 40 ) of the calibration curves. Signals were measure from the same test zones by the RGB color sensor $\left(R^{2}=0.993\right)$, a desktop scanner $\left(R^{2}=0.970\right)$, and a cellphone camera $\left(R^{2}=0.894\right)$. The $R G B$ color sensor provides higher intensity signals than the scanner and cellphone

higher (average: 3.76; Fig. S3), LOD lower, and COD higher than those of the cellphone. Compared to the desktop scanner and the cellphone, the RGB color sensor provided the highest SNR (average: 14.07; Fig. S3), the lowest LOD, and the highest COD.

The superior performance of our colorimetric reader for colorimetric measurement was due to the well-controlled illumination environment of our colorimetric reader, and more importantly, due to the transmission-based measurement mode of the colorimetric signal of the test zone. The scanner and the cellphone camera are both based on light reflection on the paper surface of the test zone, and can thus only quantify the color change on the test zone surface. In contrast, our colorimetric reader quantifies the transmitted light through the entire paper thickness of the test zone, which reflects the total color change of the test zone along its thickness. Thereby, the RGB sensor provided the highest SNR, the lowest LOD, and the highest COD. From the three calibration curves in Fig. 5b, one can also find that the RGB sensor provided the highest sensitivity among all the three methods.

\section{Autonomous sandwich ELISA for animal tissue samples}

Sandwich ELISA is a popular protocol with high sensitivity and specificity, and is more widely used for analyzing real samples with a complex protein background than direct ELISA. We also carried out sandwich ELISA of TNF- $\alpha$ in protein extraction solutions from rat laryngeal tissue, specifically vocal folds, with our platform. TNF- $\alpha$ is a cell signaling molecule that regulates the response of immune cells to injury, inflammation, and healing. Dysregulation of TNF- $\alpha$ has been associated with multiple physiological dysfunctions and diseases such as cancer ${ }^{33}$, Alzheimer's disease ${ }^{34}$, major depression ${ }^{35}$, and inflammatory bowel disease ${ }^{36}$. To demonstrate the potential clinical application of our platform, protein samples extracted from surgically injured rat vocal fold tissues ${ }^{37}$ were subjected to both conventional and $\mu \mathrm{PAD}$-based ELISAs for evaluation.

We first performed sandwich ELISA on our platform for rat TNF- $\alpha$ spiked in $1 \times$ PBS in five-fold dilutions $(19 \mathrm{pM}$ to $59 \mathrm{nM}$ ) to generate a calibration curve. Before each assay, $3 \mu \mathrm{L}$ of anti-rat TNF- $\alpha$ in PBS $(0.1 \mathrm{mg} / \mathrm{mL})$ was 
added to the potassium periodate $\left(\mathrm{KIO}_{4}\right)$-functionalized test zone as the capture antibody, and dried at room temperature for immobilization. Then, $3 \mu \mathrm{L}$ of the blocking buffer $[0.5 \%(\mathrm{v} / \mathrm{v})$ Tween -20 and $10 \%(\mathrm{w} / \mathrm{v})$ BSA in PBS] was added to the test zone and dried at room temperature to fill all the vacant sites on the test zone. After that, $3 \mu \mathrm{L}$ of rat TNF- $\alpha$ sample solution was spotted to the test zone for binding with the capture antibodies. $3 \mu \mathrm{L}$ of $1: 1(\mathrm{v} / \mathrm{v})$ mixture of biotin-conjugated anti-rat TNF- $\alpha(0.2 \mathrm{mg} / \mathrm{mL}$ in PBS; as the secondary antibody) and HRP streptavidin $(0.2 \mathrm{mg} / \mathrm{mL}$ in PBS; to in situ label the secondary antibody with HRP) was stored in the storage zone \#1 (Fig. 1a), $3 \mu \mathrm{L}$ of HRP substrate ( $4 \mathrm{mM}$ TMB in DMSO and $0.05 \mathrm{M}$ phosphate-citrate buffer with trace amount of fresh $30 \%$ hydrogen peroxide, $\mathrm{pH} 5.0$ ) in the storage zone \#2, and $3 \mu \mathrm{L}$ of stop solution (4M sulfuric acid) in the storage zone \#3. The sandwich ELISA was carried out with following steps. (i) The mixture of biotinconjugated antibodies with HRP streptavidin was transferred to the test zone, and incubated for $1 \mathrm{~min}$. (ii) the test zone was washed by PBS, and incubated for $10 \mathrm{~min}$. (iii) The HRP substrate was transferred to the test zone, and incubated for $10 \mathrm{~min}$. (iv) The stop solution was transferred to the test zone for the signal production, and incubated for $10 \mathrm{~min}$. (v) Finally, the colorimetric signal was measured. Figure 6a shows the scheme of the sandwich ELISA on our $\mu$ PAD. The measured results at different concentrations of TNF- $\alpha$ were fit into the Hill equation, as shown in Fig. 6b. The LOD of our platform for TNF- $\alpha$ detection was determined to be $22 \mathrm{pM}$. More thorough optimization of our sandwich ELISA protocol may further reduce the LOD of our platform. For confirming the effectiveness of pre-mixing the secondary antibody and HRP streptavidin and storing them in the same storage zone, we also carried out assays by sequentially adding the secondary antibody and the HRP streptavidin to the test zone as control experiments. We compared the results with these from our original protocol above through student $t$-test, and found no significant difference ( $p=0.132, n=7$; Fig. S4) between the two data sets. This confirms the effectiveness of our original protocol.

Finally, we conducted sandwich ELISA for detection of TNF- $\alpha$ in protein extractions from injured rat vocal folds 2 days $(n=4)$ and 4 weeks $(n=5)$ following surgery ${ }^{37}$. We also benchmarked our platform through testing the same samples using a commercial ELISA kit (ab100785, Abcam) on a 96-well plate. Figure 6c shows the testing results from our platform (red bars) and the commercial ELISA kit (blue bars). For the sample group of 4 weeks after surgery, the TNF- $\alpha$ concentrations $(231.6 \pm 103.09$ $\mathrm{pM})$ measured by our platform reveal no significant difference $(p=0.77)$ from those obtained by the commercial ELISA kit $(247.4 \pm 30.3 \mathrm{pM})$. For the sample group of
2 days after surgery, their TNF- $\alpha$ concentrations $(69.9 \pm$ $10.4 \mathrm{pM})$ are close to the LOD (27 pM) of our platform; thus, the measurement results from our platform become significantly lower $(p=0.003)$ than these from the standard ELISA. These results show that our platform is capable of detecting animal cytokine proteins within the normal measurement range. From the standard ELISA results in Fig. 6c, one can also observe that the TNF- $\alpha$ concentration in the vocal fold tissue extractions increased from $69.9 \mathrm{pM} 2$ days after surgery to $247.4 \mathrm{pM}$ 4 weeks after surgery, corresponding to the dual roles of TNF- $\alpha$ in the acute and later phases of wound healing ${ }^{37,38}$. To further evaluate the agreement of the two diagnostic methods based on our platform and the standard ELISA, we performed Bland-Altman analysis ${ }^{39}$ of the measurement results. As shown in Fig. 6d, the Bland-Altman analysis demonstrates a good agreement between the two methods with the mean difference $(-26.5 \mathrm{pM})$ and limits of agreement (95\% confidence interval: -151.6 to 98.6 $\mathrm{pM})$. All of the sample points located within the 95\% confidence interval around the mean. This result further demonstrates the potential use of our platform as a SIAO diagnostic platform for real diagnostic applications.

\section{Materials and methods \\ Materials and reagents}

Whatman No. 1 chromatography paper, bovine serum albumin (BSA), rabbit IgG, anti-rabbit IgG (alkaline phosphatase conjugated), anti-rabbit IgG (fluorescein isothiocyanate conjugated), 3,3',5,5'-tetramethylbenzidine (TMB) (99\%), BCIP ${ }^{\circledR} / \mathrm{NBT}$ tablets, Tween ${ }^{\circledR} 20,10 \times$ phosphate buffered saline (PBS), and potassium periodate were purchased from Sigma-Aldrich (Oakville, ON, Canada), and used as received without further purification. Recombinant rat TNF- $\alpha$, rat anti-TNF- $\alpha$ antibody, and horseradish peroxidase (HRP)-conjugated streptavidin were purchased from Abcam (Toronto, ON). Biotinylated anti-Rat TNF- $\alpha$ was purchased from BioLegend (San Diego, CA). The white LED and polyolefin (PO) were purchased from Digi-Key Cooperation (Thief River Falls, $\mathrm{MN})$. The Arduino UNO microcontroller and the $16 \times 2$ LCD were purchased from RobotShop Inc. (Mirabel, QC). The Pyralux $^{\circledR}$ (LF7062) copper-coated polymide film was obtained as sample from DuPont (Research Triangle Park, NC, USA). Ferric chloride was purchased for etching the copper from MG Chemicals (Burlington, ON, Canada). The RGB color sensor (TCS34725) was purchased from Adafruit (New York, NY, USA). The scotch plastic thermal laminating pouches were purchased from $3 \mathrm{M}$ (Milton, ON, Canada).

\section{Fabrication and preparation of the $\mu$ PAD}

The channel layer and the isolation layer of the $\mu \mathrm{PAD}$, both made from chromatography paper, were fabricated 
a
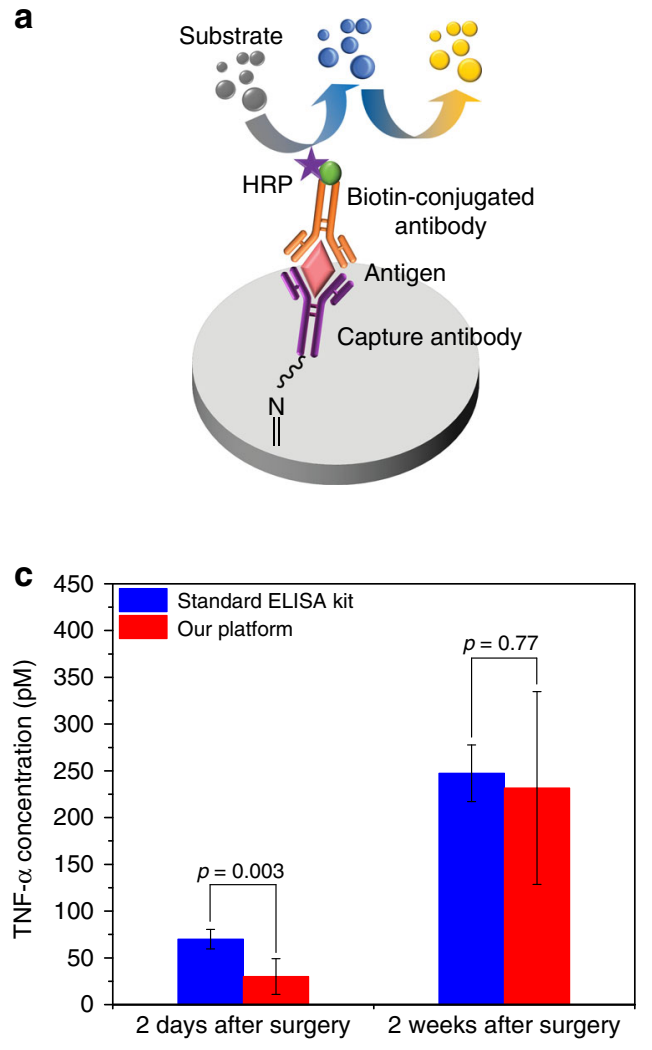

b

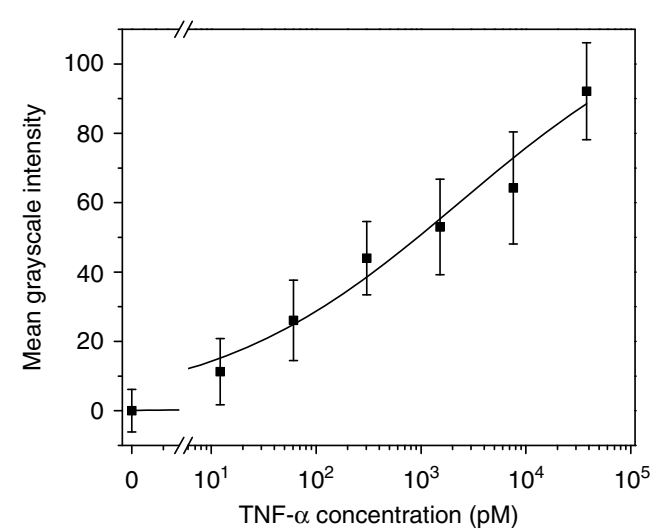

d

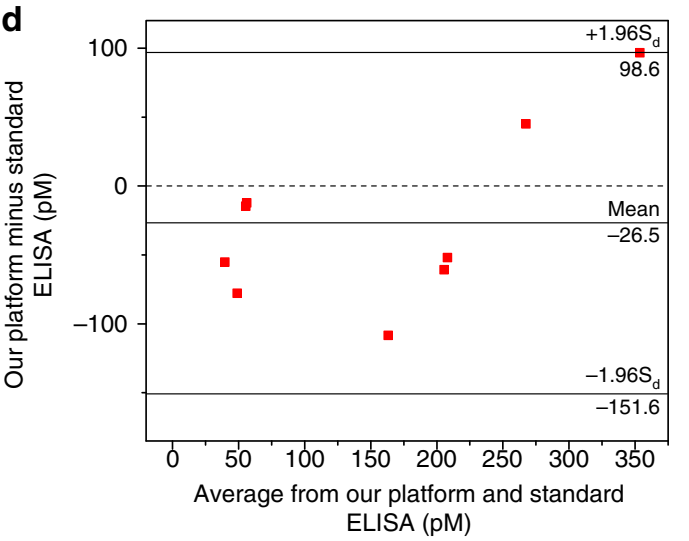

Fig. 6 Sandwich P-ELISA for TNF-a in protein extractions from surgically injured rat vocal fold tissue tested by our platform and the standard ELISA. a Schematic of a sandwich ELISA on the HPAD. HRP substrates were catalyzed by HRP into blue products. The catalyzing reaction was stopped with sulfuric acid to produce yellow color. $\mathbf{b}$ Calibration curve of the mean grayscale intensity signal measured from our platform versus the TNF-a concentration $(n=5)$. c The comparison of testing data from our platform and the standard ELISA on extraction samples of rat vocal fold tissues two days and four weeks after surgery. $\mathbf{d}$ Bland-Altman analysis on the diagnostic methods based on our platform and the standard ELISA

using wax printing and laser cutting ${ }^{40}$. To fabricate the channel layer, a Xerox 8570 inkjet printer was used to print solid wax (black) patterns on Whatman No. 1 chromatography paper to define the paper channels. The paper with solid wax printed on its surface was then placed on a hotplate at $120^{\circ} \mathrm{C}$ for $30 \mathrm{~s}$ to melt the wax and form hydrophilic channels inside the paper. After that, paper cantilever beams were cut out of the channel layer using a $\mathrm{CO}_{2}$ laser cutter (VLS 2.30, Universal Laser Systems). The root of each paper cantilever was cut using dashed lines to form a foldable hinge, and the free end of each paper cantilever was cut to disconnect the test zone and a reagent storage zone (Fig. 2b). A hydrophilic paper bridge was cut out of soft tissue paper (Delicate Task Wipers, Kimwires) and attached to the free end of the paper cantilever valve. This paper bridge connects the test zone and the storage zone when the paper valve is driven to its on-state position (Fig. 2c). The isolation layer was constructed by fully impregnating Whatman No. 1 chromatography paper with solid wax (through wax printing and $120^{\circ} \mathrm{C}$ baking) and then laser-cutting it to form $6 \mathrm{~mm} \times 12 \mathrm{~mm}$ windows with their positions aligned with the ones of the heating resistors (on the heating PCB) and the SMP actuators (on the channel layer). The channel layer and the isolation layer were bonded together using $3 \mathrm{M}$ Scotch double-sided tape. The hydrophobic isolation layer allows efficient heat transfer from the heating resistor to the SMPs, and in the meanwhile, eliminates any fluid leakage from the channel layer to the heater layer (Fig. 3b).

To carry out an ELISA, proteins always need to be immobilized onto the surfaces of cellulose microfibers of the test zone. We treated the paper test zone through aldehyde functionalization (Fig. S5). The test zone was baked at $65^{\circ} \mathrm{C}$ and then spotted with $3 \mu \mathrm{L}$ of $\mathrm{KIO}_{4}$ aqueous solution $(0.031 \mathrm{M}$ and $\mathrm{pH}=5)$ for oxidation, and the spotting was repeated every $5 \mathrm{~min}$ for $2 \mathrm{~h}$. After oxidization, $10 \mu \mathrm{L}$ of deionized water $\left(\mathrm{diH}_{2} \mathrm{O}\right)$ was added for washing away the residual oxide, and the washing process was repeated twice. Finally, the paper was dried in a 
desiccator for at least $12 \mathrm{~h}$ before use. The aldehyde groups created on the cellulose skeleton of the paper test zone can covalently immobilize proteins containing amino groups through the Schiff-base linkage ${ }^{41}$. The effectiveness of the oxidization process was confirmed by Fourier-transform infrared spectroscopy (FTIR). From the FTIR spectrum of the $\mathrm{KIO}_{4}$-modifed paper surface (Fig. S6), the characteristic absorption band of aldehyde group on the paper surface appeared at $1726 \mathrm{~cm}^{-1}$ due to the stretching vibration of the $\mathrm{C}=\mathrm{O}$ double bond ${ }^{42}$.

Before each assay, reagent solutions were added to their corresponding storage zones of a $\mu \mathrm{PAD}$, and dried at room temperature for $10 \mathrm{~min}$. To determine the amount of reagent that needs to be stored in the test zone, we tested the reagent transfer efficiency from the storage zone to the test zone using fluorophore-tagged antibody as a tracking reagent. Note all the storage zones and their downstream channels connecting the test zone are identical. $3 \mu \mathrm{L}$ of FITC-conjugated rabbit IgG antibody (antirabbit IgG; $0.1 \mathrm{mg} / \mathrm{mL}$ ) was transferred from the storage zone to the test zone, and the continuous transfer flow of the PBS buffer lasted for $45 \mathrm{~s}$. By measuring the fluorescence intensity of the storage zone before and after the transfer, we found that $\sim 10 \%$ of the stored FITCconjugated anti-rabbit IgG was eventually delivered to the test zone. Thus, the concentrations of the reagent solutions added to the storage zone was determined to be ten times the regular concentrations of the reagents used in a previously reported ELISA protocol on $\mu \mathrm{PAD}^{3}$.

Finally, a plastic lamination layer, which was laser-cut out of 0.127-mm-thick plastic film (Thermal Laminating Pouches TP5903-20, 3M), was attached to the top surface of the channel layer using an impulse heat sealer (PFS100, Goplus). Four lamination lines (Fig. 3a) were applied to the peripheral areas of the channel layer to bond the plastic lamination layer and the channel layer. This line bonding ensures that the thermal lamination does not impact the activity of the stored reagents.

\section{Conclusion}

We developed a novel SMP-actuated, controllable fluid valve for fluid manipulation on a $\mu \mathrm{PAD}$, which enabled a paper-based microfluidic platform for automated, multistep ELISA. The merits of this new platform are summarized as follows. (i) The first SMP-actuated, on-chip paper valve for automatic fluid manipulation: The valve is activated by localized Joule heating, and its relatively small footprint allows the integration of multiple valves onto a single $\mu \mathrm{PAD}$ to run multi-step assays. Thanks to the dualstate operation of the SMP actuator, the valve can be turned on and off using the sample heating resistor, and the length of its on-state period can be readily regulated. (ii) A new single-layer $\mu$ PAD design with several SMPactuated valves for automated multi-step ELISA. The $\mu \mathrm{PAD}$ design leverages valve-regulated fluid transfer to enable automated operation of multiple assay steps involving reagent addition, incubation, washing, and signal amplification. (iii) A sensitive light-transmittancebased mechanism for colorimetric signal detection on a $\mu \mathrm{PAD}$. We demonstrated that, for the same readout signal on our single-layer $\mu \mathrm{PAD}$, our colorimetric reader, which measured the light transmittance of the paper test zone, provided better LOD and COD of the calibration curve than a desktop scanner and a cellphone camera. In addition, our signal measurement setup only employed a pair of LED and RGB sensor mounted on the colorimetric reader, which is a more cost-efficient and integrated solution than a separate measurement device. (iv) A novel light-transmittance-based mechanism for valve malfunction detection. The operation success rate of the SMPactuated valves was determined to be $97 \%$. Based on optical measurement of the wetting condition of the paper test zone at different operation steps, this mechanism reliably monitored the operations of all the on-chip valves and flagged any valve malfunction to completely eliminate the malfunction-induced measurement errors. (v) An integrated, user-friendly design of the diagnostic platform, including the $\mu \mathrm{PAD}$ and the colorimetric reader, to realize automated ELISA operation, valve malfunction monitoring, final data collection, and wireless data transmission, if desired.

Although multi-step ELISA was demonstrated on our platform, the designs of the $\mu \mathrm{PAD}$ and the entire platform can be readily extended to implement other types of multi-step diagnostic assays in a SIAO fashion, and are suitable for rapid diagnostic tests at the POC or in any settings where sophisticated equipment and skilled personnel are not available. Examples of other colorimetric assays our platform can be applied to include: glucose $\mathrm{e}^{2}$, $\operatorname{protein}^{43}$, uric $\operatorname{acid}^{44}$, lactate ${ }^{45}, \mathrm{pH}^{46}$, and pathogenic bacteria $^{47,48}$.

\footnotetext{
Acknowledgements

We gratefully acknowledge the support from the Natural Sciences and Engineering Research Council of Canada (grant numbers: RGPIN-06374-2017, RGPAS-507980-2017) and National Institute on Deafness and Other Communication Disorders (grant number: R03DC012112), and from the Canadian Institutes of Health Research (grant number: PJT-159475). We also acknowledge the financial supports from the Canada Research Chairs Program (grand number: 950-231265) to N.Y.K.L.-J., from the Chinese Scholarship Council to P.S., Q.W. and P.P.
}

\section{Author details}

${ }^{1}$ Department of Mechanical and Industrial Engineering, University of Toronto, Toronto, ON, M5S 3G8, Canada. 'Department of Mechanical Engineering, McGill University, Montreal, QC, H3A OC3, Canada. ${ }^{3}$ Department of Electrical and Electronic Engineering, Xi'an Jiaotong-Liverpool University, 215123 Suzhou, Jiangsu, China. ${ }^{4}$ School of Communication Sciences and Disorders, McGill University, Montreal, QC H3A 1G1, Canada. Institute of Biomaterials and Biomedical Engineering, University of Toronto, Toronto, ON M5S 3G9, Canada. ${ }^{6}$ Present address: Department of Chemistry, Stanford University, Stanford, CA 94305, USA 


\section{Authors' contributions}

H.F. and X.L. conceived of the idea of this work and designed the experiments. H.F. designed and fabricated the microfluidic platform, performed the experiments of immunoassays and collected the experimental data. P.S. carried out the protocol optimization experiments and collected the data. Q.W. and C.Z. wrote the code for the platform automation and Bluetooth communication. P.P. and X.L. fabricated the colorimetric reader and the $\mu$ PADs for experiments. N.Y.K.L.-J. provided the animal specimens and data. H.F., P.S., and X.L. wrote the manuscript. All authors contributed to the general discussion and revision of the manuscript.

\section{Conflict of interest}

The authors declare that they have no conflict of interest.

Supplementary Information accompanies this paper at https://doi.org/ 10.1038/s41378-019-0091-0.

Received: 1 February 2019 Revised: 31 May 2019 Accepted: 10 June 2019 Published online: 23 September 2019

\section{References}

1. Martinez, A. W., Phillips, S. T., Whitesides, G. M. \& Carrilho, E. Diagnostics for the developing world: microfluidic paper-based analytical devices. Anal. Chem. 82, 3-10 (2009).

2. Martinez, A. W., Phillips, S. T., Butte, M. J. \& Whitesides, G. M. Patterned paper as a platform for inexpensive, low-volume, portable bioassays. Angew. Chem. Int. Ed. 46, 1318-1320 (2007).

3. Cheng, C. M. et al. Paper-based ELISA. Angew. Chem. Int. Ed. 49, 4771-4774 (2010).

4. Liu, X. et al. in 2011 IEEE 24th International Conference on Micro Electro Mechanical Systems (MEMS) 75-78 (IEEE, 2011).

5. Yamada, K., Takaki, S., Komuro, N., Suzuki, K. \& Citterio, D. An antibody-free microfluidic paper-based analytical device for the determination of tear fluid lactoferrin by fluorescence sensitization of Tb3+. Analyst 139, 1637-1643 (2014).

6. Zang, D., Ge, L., Yan, M., Song, X. \& Yu, J. Electrochemical immunoassay on a 3D microfluidic paper-based device. Chem. Commun. 48, 4683-4685 (2012).

7. Li, L. et al. Paper-based electrochemiluminescence immunodevice for carcinoembryonic antigen using nanoporous gold-chitosan hybrids and graphene quantum dots functionalized Au@Pt. Sens. Actuators B Chem. 202, 314-322 (2014).

8. Li, X. \& Liu, X. A microfluidic paper-based origami nanobiosensor for label-free, ultrasensitive immunoassays. Adv. Healthc. Mater. 5, 1326-1335 (2016).

9. Li, X., Tian, J., Nguyen, T. \& Shen, W. Paper-based microfluidic devices by plasma treatment. Anal. Chem. 80, 9131-9134 (2008).

10. Liu, H., Li, X. \& Crooks, R. M. Paper-based SlipPAD for high-throughput chemical sensing. Anal. Chem. 85, 4263-4267 (2013).

11. Martinez, A. W. et al. Programmable diagnostic devices made from paper and tape. Lab Chip 10, 2499-2504 (2010).

12. Glavan, A. C. et al. Rapid fabrication of pressure-driven open-channel microfluidic devices in omniphobic RF paper. Lab Chip 13, 2922-2930 (2013).

13. Fu, E., Kauffman, P., Lutz, B. \& Yager, P. Chemical signal amplification in twodimensional paper networks. Sens. Actuat. B Chem. 149, 325-328 (2010).

14. Fu, E. et al. Two-dimensional paper network format that enables simple multistep assays for use in low-resource settings in the context of malaria antigen detection. Anal. Chem. 84, 4574-4579 (2012)

15. Chen, H., Cogswell, J., Anagnostopoulos, C. \& Faghri, M. A fluidic diode, valves, and a sequential-loading circuit fabricated on layered paper. Lab Chip 12, 2909-2913 (2012).

16. Gerbers, R., Foellscher, W., Chen, H., Anagnostopoulos, C. \& Faghri, M. A new paper-based platform technology for point-of-care diagnostics. Lab Chip 14, 4042-4049 (2014).

17. Chen, H., Faghri, M. \& Anagnostopoulos, C. in 2014 IEEE Healthcare Innovation Conference (HIC) 304-306 (IEEE, 2014).

18. Koo, C. K. He, F. \& Nugen, S. R. An inkjet-printed electrowetting valve for paper-fluidic sensors. Analyst 138, 4998-5004 (2013).
19. Houghtaling, J., Liang, T., Thiessen, G. \& Fu, E. Dissolvable bridges for manipulating fluid volumes in paper networks. Anal. Chem. 85, 11201-11204 (2013).

20. Jahanshahi-Anbuhi, S. et al. Paper-based microfluidics with an erodible polymeric bridge giving controlled release and timed flow shutoff. Lab Chip 14 229-236 (2014).

21. Lafleur, L. K. et al. A rapid, instrument-free, sample-to-result nucleic acid amplification test. Lab Chip 16, 3777-3787 (2016).

22. Phillips, E. A., Shen, R., Zhao, S. \& Linnes, J. C. Thermally actuated wax valves for paper-fluidic diagnostics. Lab Chip 16, 4230-4236 (2016).

23. Toley, B. J. et al. Tunable-delay shunts for paper microfluidic devices. Anal. Chem. 85, 11545-11552 (2013).

24. Giokas, D. L., Tsogas, G. Z. \& Vlessidis, A. G. Programming fluid transport in paper-based microfluidic devices using razor-crafted open channels. Anal. Chem. 86, 6202-6207 (2014).

25. Salentijn, G. I., Hamidon, N. \& Verpoorte, E. Solvent-dependent on/off valving using selectively permeable barriers in paper microfluidics. Lab Chip 16, 1013-1021 (2016).

26. Fobel, R., Kirby, A. E., Ng, A. H., Farnood, R. R. \& Wheeler, A. R. Paper microfluidics goes digital. Adv. Mater. 26, 2838-2843 (2014).

27. Verma, M. S. et al. Sliding-strip microfluidic device enables ELISA on paper. Biosens. Bioelectron. 99, 77-84 (2018).

28. Li, X., Zwanenburg, P. \& Liu, X. Magnetic timing valves for fluid control in paper-based microfluidics. Lab Chip 13, 2609-2614 (2013).

29. Fratzl, M. et al. Magnetic two-way valves for paper-based capillary-driven microfluidic devices. ACS Omega 3, 2049-2057 (2018).

30. Toley, B. J. et al. A versatile valving toolkit for automating fluidic operations in paper microfluidic devices. Lab Chip 15, 1432-1444 (2015).

31. Felton, S. M. et al. Self-folding with shape memory composites. Soft Matter $\mathbf{9}$, 7688-7694 (2013)

32. Felton, S., Tolley, M., Demaine, E., Rus, D. \& Wood, R. A method for building self-folding machines. Science 345, 644-646 (2014).

33. Locksley, R. M., Killeen, N. \& Lenardo, M. J. The TNF and TNF receptor superfamilies: integrating mammalian biology. Cell 104, 487-501 (2001).

34. Swardfager, W. et al. A meta-analysis of cytokines in Alzheimer's disease. Biol. Psychiat. 68, 930-941 (2010).

35. Dowlati, Y. et al. A meta-analysis of cytokines in major depression. Biol. Psychiat 67, 446-457 (2010).

36. Brynskov, J. et al. Tumour necrosis factor a converting enzyme (TACE) activity in the colonic mucosa of patients with inflammatory bowel disease. Gut 51, 37-43 (2002)

37. Li-Jessen, N. Y., Powell, M., Choi, A. J., Lee, B. J. \& Thibeault, S. L. Cellular source and proinflammatory roles of high-mobility group box 1 in surgically injured rat vocal folds. Laryngoscope 127, E193-E200 (2017).

38. Chi, L.-Y. et al. The dual role of tumor necrosis factor-alpha in the pathophysiology of spinal cord injury. Neurosci. Lett. 438, 174-179 (2008).

39. Giavarina, D. Understanding bland altman analysis. Biochem. Med. 25, 141-151 (2015).

40. Li, X. \& Liu, X. Fabrication of three-dimensional microfluidic channels in a single layer of cellulose paper. Microfluid. Nanofluid. 16, 819-827 (2014).

41. Badu-Tawiah, A. K. et al. Polymerization-based signal amplification for paperbased immunoassays. Lab Chip 15, 655-659 (2015).

42. Liu, X., Nishi, N., Tokura, S. \& Sakairi, N. Chitosan coated cotton fiber: preparation and physical properties. Carbohydr. Polym. 44, 233-238 (2001).

43. Martinez, A. W., Phillips, S. T. \& Whitesides, G. M. Three-dimensional microfluidic devices fabricated in layered paper and tape. Proc. Natl Acad. Sci. USA 105 , 19606-19611 (2008)

44. Li, X., Tian, J. \& Shen, W. Progress in patterned paper sizing for fabrication of paper-based microfluidic sensors. Cellulose 17, 649-659 (2010).

45. Dungchai, W., Chailapakul, O. \& Henry, C. S. Use of multiple colorimetric indicators for paper-based microfluidic devices. Anal. Chim. Acta 674, 227-233 (2010).

46. Abe, K., Suzuki, K. \& Citterio, D. Inkjet-printed microfluidic multianalyte chemical sensing paper. Anal. Chem. 80, 6928-6934 (2008).

47. Li, C.-z et al. Paper based point-of-care testing disc for multiplex whole cell bacteria analysis. Biosens. Bioelectron. 26, 4342-4348 (2011).

48. Jokerst, J. C. et al. Development of a paper-based analytical device for colorimetric detection of select foodborne pathogens. Anal. Chem. 84, 2900-2907 (2012) 